\title{
Celastrol suppresses the proliferation of lung adenocarcinoma cells by regulating microRNA-24 and microRNA-181b
}

\author{
YUN-FEI YAN $^{1 *}$, HAN-HAN ZHANG $^{1 *}$, QING LV $^{1}$, YUE-MEI LIU ${ }^{1}$, YOU-JIE LI ${ }^{1}$, \\ BAO-SHENG LI ${ }^{2}$, PING-YU WANG ${ }^{1}$, WEN-JING SHANG ${ }^{1}$, ZHEN YUE $^{1}$ and SHU-YANG XIE ${ }^{1}$ \\ ${ }^{1}$ Department of Biochemistry and Molecular Biology, Key Laboratory of Tumor Molecular Biology, \\ Binzhou Medical University, Yantai, Shandong 264003; ${ }^{2}$ Department of Radiation Oncology, Shandong \\ Cancer Hospital, Shandong Academy of Medical Sciences, Jinan, Shandong 250117, P.R. China
}

Received January 6, 2016; Accepted May 26, 2017

DOI: $10.3892 / \mathrm{ol} .2017 .7593$

\begin{abstract}
Cumulative evidence has indicated that celastrol may suppress cancer growth; however, the underlying mechanism requires further investigation. In the present study, A549 cells were treated with various concentrations of celastrol. Lung cancer cell proliferation was evaluated using an MTT assay and observed under a microscope. Cell apoptosis was detected by Annexin V fluorescein isothiocyanate/propidium iodide double-labeled flow cytometry. The results demonstrated that celastrol suppressed proliferation and induced apoptosis in a dose-independent manner. Celastrol may also decrease the phosphorylation levels of signal transducer and activator of transcription 3 (STAT3) and the B cell lymphoma-2 (Bcl-2)/Bcl-2 associated $\mathrm{C}$ protein (Bax) ratio. As microRNA (miR-24 and miR-181b) were predicated to target STAT3, STAT3 activation was inhibited in miR-24-or miR-181b-treated A549 cells compared with the control treatment. The ratio of Bcl-2/Bax was further reduced in miR-24 or miR-181b-treated A549 cells. The results were further confirmed by detecting in another lung adenocarcinoma cell line, LTEP-a-2. In summary, the results of the present study demonstrated that celastrol treatment suppressed the proliferation and induced apoptosis by regulating the expression levels of miR-24 and miR-181b.
\end{abstract}

\section{Introduction}

Celastrol is a pharmacologically active compound that was originally isolated from Thunder God Vine

Correspondence to: Dr Shu-Yang Xie, Department of Biochemistry and Molecular Biology, Key Laboratory of Tumor Molecular Biology, Binzhou Medical University, Yantai, Shandong 264003, P.R. China E-mail: shuyangxie@aliyun.com

*Contributed equally

Key words: celastrol, signal transducer and activator of transcription 3, microRNA-24, microRNA-181b, cell apoptosis
(Tripterygium wilfordii; traditional Chinese medicine). Celastrol was identified to be a pentacyclic triterpenoid, belonging to a small category of natural products of triterpene quinine methides $(1,2)$. Containing electrophilic sites within the rings of quinone methide structure, celastrol is able to form covalent Michael adducts with the nucleophilic thiol groups of cysteine residues $(3,4)$. This appears to be the mechanism underlying celastrol-mediated effects on the functions of various proteins, although the structural determinants in proteins can also regulate their interaction with celastrol and the covalent adducts formation (5).

Numerous previous studies have indicated that celastrol may protect against a variety of inflammatory diseases in animal models $(6,7)$. Furthermore, celastrol is a promising anticancer drug, which can suppress the proliferation of various cancer cells and prevent their malignant tissue invasion and obstruct angiogenesis (8-10). Certain therapeutic studies also demonstrated that celastrol can sensitize resistant melanoma cell to temozolomide treatment and potentiate radiotherapy of prostate cancer cells in combination therapy $(11,12)$.

Signal transducer and activator of transcription 3 (STAT3) has been identified as a key molecular target of celastrol $(13,14)$; however, the mechanism underlying the effects of celastrol on STAT3 remains to be elucidated. In therapeutic investigations of celastrol, certain studies suggest that celastrol may induce apoptosis and repress invasion of cancer cells by regulation of microRNA (miRNA) expression levels $(15,16)$. Therefore, the present study investigated whether the STAT3 signaling pathway is associated with the expression levels of miRNAs. The present study further investigated whether celastrol inhibited lung cancer apoptosis via STAT3-associated miRNAs.

\section{Materials and methods}

Cell culture. A549 human lung adenocarcinoma cells purchased from Shanghai Institute of Pharmaceutical Industry (Shanghai, China) were cultured in RPMI-1640 supplemented with $10 \%$ fetal bovine serum (both from Gibco; Thermo Fisher Scientific, Inc., Waltham, MA, USA) and $100 \mathrm{U} / \mathrm{ml}$ penicillin-streptomycin (Sigma-Aldrich; Merck KGaA, Darmstadt, Germany) and maintained at $37^{\circ} \mathrm{C}$ in a humidified incubator containing $5 \% \mathrm{CO}_{2}$. Cells $\left(5 \times 10^{5}\right)$ in the logarithmic growth phase were 
seeded in 6-well plates, and celastrol (Sigma-Aldrich; Merck $\mathrm{KGaA}$ ) was dissolved in dimethyl sulfoxide (DMSO) and added to the plate in complete RPMI-1640 medium when the cell confluence arrived at 70\%. Cells were incubated for $48 \mathrm{~h}$ at $37^{\circ} \mathrm{C}$. LTEP-a-2, another human lung adenocarcinoma cell line also came from Shanghai Institute of Pharmaceutical Industry, were used under the same culture condition as A549 cells.

MTT assay. Cells $\left(1 \times 10^{4}\right)$ were cultured into 96-well plates. Various concentrations of celastrol $(0,1.5,3$ and $4.5 \mu \mathrm{M})$ were added to the medium and 6 replicates were performed for each concentration. Cells were cultivated for $48 \mathrm{~h}$ at $37^{\circ} \mathrm{C}$, MTT assays were performed to determine the cell viability and the growth inhibition rate of A549 cells. A total of $10 \mu \mathrm{l}$ MTT $(5 \mathrm{mg} / \mathrm{ml})$ was added to each well and the supernatant was removed by pipette after another $4 \mathrm{~h}$ of incubation at $37^{\circ} \mathrm{C}$. Subsequently, $100 \mu 1$ DMSO (Sigma-Aldrich; Merck KGaA) was added to dissolve the crystals produced by MTT at room temperature for $10 \mathrm{~min}$. The optical density (OD) value was determined using an ELISA reader (ELx800; Bio-Tek Instruments, Inc., Winooski, VT, USA) at $570 \mathrm{~nm}$. Cell growth inhibition rate $=(\mathrm{OD}$ control $-\mathrm{OD}$ sample $) / \mathrm{OD}$ control $\mathrm{x} 100$ (\%) $(17,18)$.

Detection of apoptosis. Cells were cultured into 6-well plates and celastrol was added to the plate following the aforementioned concentration gradient when the cell confluence was $70 \%$ on the following day. Detection of apoptosis was performed after $48 \mathrm{~h}$ incubation at $37^{\circ} \mathrm{C}$, according to the Annexin V-fluorescein isothiocyanate (FITC)/propidium iodide (PI) apoptosis detection kit protocol (BD Biosciences, Franklin Lakes, NJ, USA). Firstly, A549 cells were gently washed with PBS twice. Subsequently, the cells were centrifuged for $5 \mathrm{~min}$ at $650 \mathrm{x} \mathrm{g}$ at room temperature, the supernatant was discarded and $100 \mu 1 \mathrm{X}$ binding buffer was added to each tube for re-suspension at room temperature. Next, $5 \mu \mathrm{l}$ annexin V-FITC and $5 \mu \mathrm{l}$ PI were added and cells were incubated at room temperature for $15 \mathrm{~min}$. The cells were then analyzed using a flow cytometer (Beckman Coulter, Inc., Brea, CA, USA).

Reverse transcription-quantitative polymerase chain reaction (RT-qPCR). A549 cells were treated with the effective concentration of celastrol $(3 \mu \mathrm{M})$ for $48 \mathrm{~h}$ at $37^{\circ} \mathrm{C}$ and the control group was established using an equivalent concentration of DMSO. miRNA from these cells was isolated using RNAiso (Takara Bio, Inc., Otsu, Japan) according to the manufacturer's instruction. Subsequently, poly(A) was added using poly(A) polymerase (Ambion; Thermo Fisher Scientific, Inc.). miRNA extraction and adding poly(A) should follow the respective protocols. Complementary DNA was synthesized using RT primer 5'-AACATGTACAGTCCATGGATGd(T) 30(A, G, C or T)-3'. miR primers were as follows: miR-24 forward, 5'-CTCCGGTGCCTACTGAGCTGA-3' and reverse, 5'-AAC ATGTACAGTCCATGGATG-3'; miR-181b forward, 5'-GGT CACAATCAACATTCATTG-3' and reverse, 5'-AACATG TACAGTCCATGGATG-3'. Human 5S rRNA was used as reference gene, and primers were as follows: Forward, 5'-GCC ATACCACCCTGAACG-3' and reverse, 5'-AACATGTAC
AGTCCATGGATG-3'. SYBR ${ }^{\circledR}$ Premix Ex Taq ${ }^{\mathrm{TM}}$ kit (Takara Bio, Inc.) was used according to the manufacturer's instructions. The expression levels of miRNA was assessed using the RG3000 system (Qiagen, Inc., Valencia, CA, USA) as follows: Initiation with $3 \mathrm{~min}$ of denaturation at $95^{\circ} \mathrm{C}$, followed by 40 cycles of amplification with $20 \mathrm{sec}$ of denaturation at $95^{\circ} \mathrm{C}, 20 \mathrm{sec}$ at $56^{\circ} \mathrm{C}$ for annealing and $20 \mathrm{sec}$ of extension at $72^{\circ} \mathrm{C}$. Fluorescence was detected at $585 \mathrm{~nm}$ using the RG3000 system (Qiagen, Inc.). The above process was repeated 3 times in triplicate. The results ware quantified using the $2^{-\Delta \Delta C q}$ method as before (19).

Western blotting. Cells were lysed using cold lysis buffer (RIPA; Beyotime Institute of Biotechnology, Haimen, China) for $30 \mathrm{~min}$ on ice and protein concentration of cell lysis was determined by a BCA assay (cat no. PC0020; Beijing Solarbio Science \& Technology Co., Ltd., Beijing, China). The experiment followed the manufacturer's instructions. Subsequently, each $35 \mu \mathrm{g}$ protein sample was subjected to SDS-PAGE (12\% separating gel, 5\% concentrating gel) for electrophoresis and transferred to polyvinylidene difluoride membranes. Following transfer, membranes were incubated in a blocking buffer (5\% non-fat milk TBST solution, TBST: $\mathrm{NaCl} 0.8 \%$, $0.02 \% \mathrm{KCl}$, Tris-base $0.3 \%$, Tween-20 $0.1 \%, \mathrm{pH} 7.4$ ) for $2 \mathrm{~h}$ at room temperature. Subsequently, the membranes were washed 3 times with TBST (10 min each time). Next, the membranes were immunoblotted with antibodies against p-STAT-3 (1:800; sc-81523) and STAT-3 (1:800; sc-8019) (both from Santa Cruz Biotechnology, Inc., Dallas, TX, USA), Bcl-2 (1:500; BS1031), BAX (1:500; BS2538) (both from Bioworld Technology, Inc., St. Louis Park, MN, USA) and GAPDH (1:3,000; TA309157; Beijing Zhongshan Golden Bridge Biotechnology Co., Ltd., Beijing, China), which was used as the control. Immunoblotting was for $16-18 \mathrm{~h}$ at $4^{\circ} \mathrm{C}$ and then washing was performed with TBST 3 times (10 min each time). Membranes were then incubated with secondary antibodies conjugated to horseradish peroxidase (1:3000; BS13271 and BS12471; Bioworld Technology, Inc.). Blots were quantified densitometrically using Quantity One software (V4.62; Bio-Rad Laboratories, Inc., Hercules, CA, USA).

miRNAs prediction. The webpage (http://www.microrna. org/microrna/home.do) was opened, input the name of protein of interest was inputted interested in the item 'Target mRNA' and miRNAs with potential interaction with the protein and could be confirmed by RT-qPCR were searched for.

Statistical analysis. Quantitative results are presented as the mean \pm standard deviation. Comparisons of parameters between the two groups were made using an unpaired Student's t-test or a Mann-Whitney U test. Statistical significance was evaluated with GraphPad Prism 5 (GraphPad Software, Inc., La Jolla, CA, USA). P $<0.05$ was considered to indicate a statistically significant difference.

\section{Results}

Celastrol inhibits proliferation and promotes apoptosis of A549 cells. To investigate the effects of celastrol on A549 cell growth, A549 cells were treated with various concentrations 
A

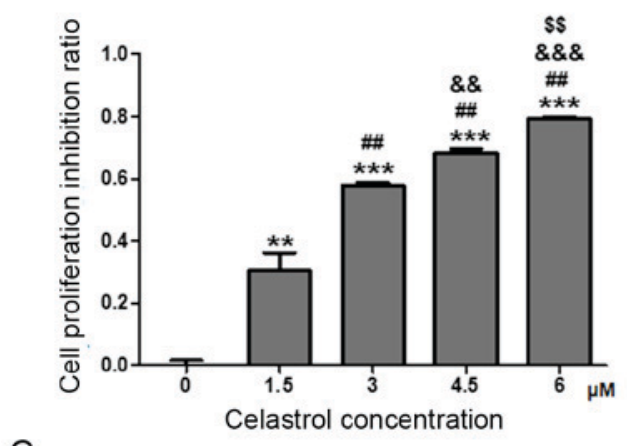

C

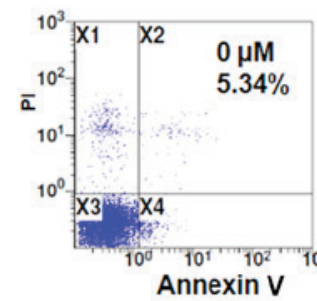

B
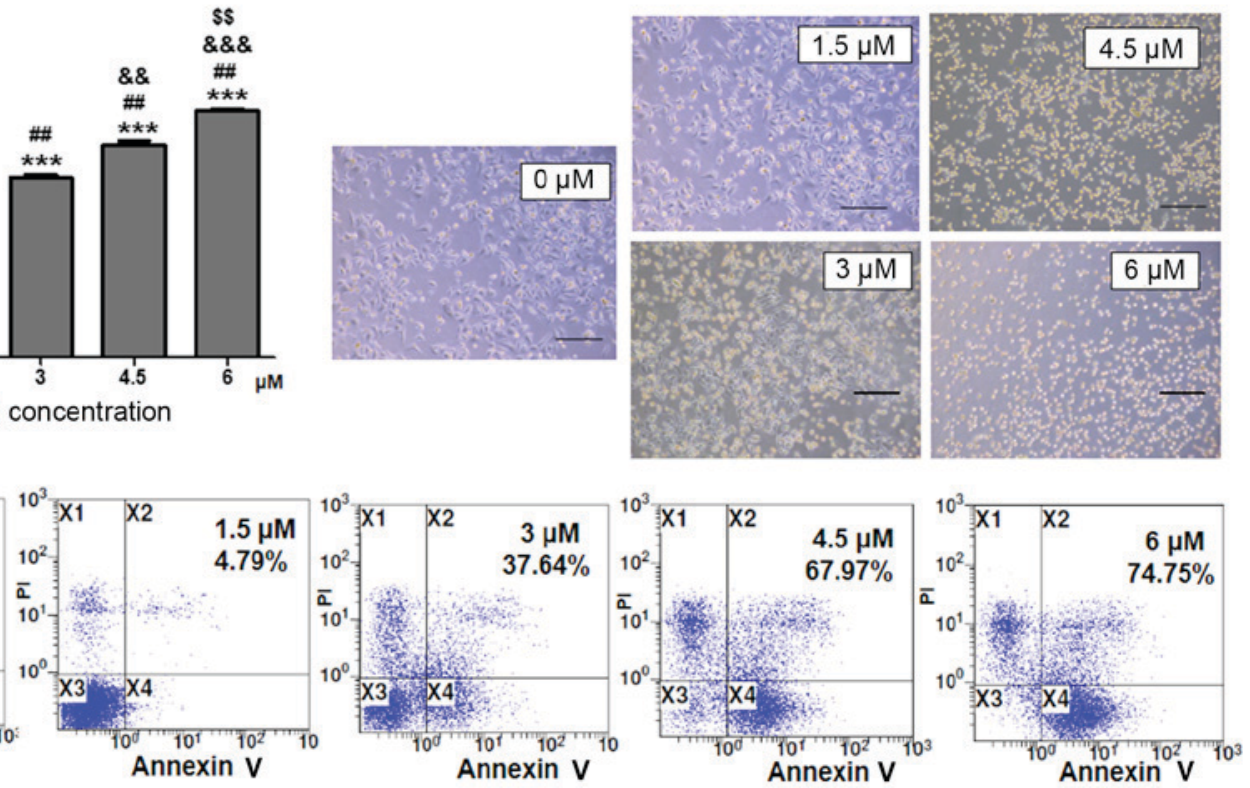

Figure 1. Celastrol suppresses proliferation and promotes apoptosis of A549 cells. (A) MTT assay investigating the proliferation of A549 cells incubated with celastrol at various concentrations. ${ }^{* *} \mathrm{P}<0.01 ;{ }^{* * *} \mathrm{P}<0.001$ vs. $0 \mu \mathrm{M} .{ }^{\# \#} \mathrm{P}<0.01$ vs. $1.5 \mu \mathrm{M}$. ${ }^{\&} \mathrm{P}<0.01$ and ${ }^{\& \& \&} \mathrm{P}<0.001$ vs. $3 \mu \mathrm{M}$. ${ }^{\$ \$} \mathrm{P}<0.01$ vs. $4.5 \mu \mathrm{M}$. Higher concentration levels of celastrol were more effective in suppressing the proliferation of A549 cells. $\mathrm{n}=6$ replicates. (B) Microscopic analysis of A549 cells following treatment with celastrol of various concentrations. Scale bar $=100 \mu \mathrm{m}$. Higher concentration of celastrol induced markedly increased levels of cell death. (C) Flow cytometry analysis. Annexin V-fluorescein isothiocyanate/propidium iodide staining was performed to evaluate the apoptosis of A549 cells. Apoptotic cells are presented in the upper left, upper right and lower right quadrants of each panel. Apoptotic cells increased with the concentration elevation of celastrol.

of celastrol for $24 \mathrm{~h}$. The MTT assay indicated that A549 cell proliferation was suppressed by celastrol in a dose-dependent manner. There was a higher level of proliferation suppression of A549 cells with a higher concentration of celastrol (Fig. 1A). The microcopy examination results confirmed these findings. There were more dead and floating cells in the higher celastrol concentration-treated cultures compared with other cultures (Fig. 1B). In order to determine whether the decrease of cell number was due to apoptosis induced by celastrol, Annexin V-FITC and PI staining was performed. The results revealed that celastrol induced A549 cell apoptosis in a dose-dependent manner (Fig. 1C). In the concentration cascade, $3 \mu \mathrm{M}$ celastrol was the closest to ID50, thus $3 \mu \mathrm{M}$ celastrol was selected as the most reasonable concentration in this study.

Celastrol decreases STAT3 phosphorylation and Bcl-2/Bax ratio. Previous studies have revealed the role of celastrol in the prevention of cancer growth, in which numerous targets of celastrol were identified, including STAT3 $(2,9,10,17)$. To further investigate the role of celastrol in lung cancer, STAT3 expression was detected in A549 cells following treatment with various concentrations of celastrol. The results demonstrated that $3 \mu \mathrm{M}(\mathrm{P}<0.0001)$ and $4.5 \mu \mathrm{M}(\mathrm{P}=0.0003)$ celastrol treatment significantly reduced STAT3 phosphorylation in A549 cells compared with $1.5 \mu \mathrm{M}$ treatment (Fig. 2A).

STAT3 serves a critical role in cell proliferation and survival. It has been revealed that deactivation of STAT3 abrogates the anti-apoptotic advantage (such as via radioresistance and chemoresistance) in various types of tumor cell lines, mainly due to the attenuation of $\mathrm{Bcl}-2(18,20)$.
Deactivation of the STAT3 signaling pathways by celastrol induced tumor cell apoptosis (Fig. 1C). The Bcl-2/Bax ratio was significantly decreased in $3 \mu \mathrm{M}(\mathrm{P}<0.0001)$ and $4.5 \mu \mathrm{M}$ $(\mathrm{P}<0.0001)$ celastrol-treated cells compared with 0 and $1.5 \mu \mathrm{M}$ celastrol-treated groups (Fig. 2B). Indeed, $4.5 \mu \mathrm{M}$ $\mathrm{P}=0.006)$ celastrol treatment also could lead $\mathrm{Bcl}-2 / \mathrm{Bax}$ ratio decreased compared with $3 \mu \mathrm{M}$ treatment (Fig. 2B). These results further suggested higher concentration celastrol treatment attenuated Bcl-2/Bax ratio more within certain scope. The apoptosis induced by celastrol may account for deactivation of the STAT3 signaling pathway.

Celastrol induces the overexpression of miR-24 and $m i R-181 b$. The molecular mechanisms underlying celastrol induction of apoptosis require further elucidation. Therefore the mechanisms underlying the effect of celastrol on STAT3 phosphorylation was investigated. Previous studies have revealed that miRNAs are involved in the antitumor mechanism underlying celastrol $(15,16)$. Therefore, the present study investigated whether celastrol induced A549 cell apoptosis through the regulation of STAT3-associated miRNAs.

Using microRNA.org software (http://www.microrna. org/microrna/home.do), the present study predicted that the 3' untranslated region (UTR) of STAT3 mRNA was targeted by miR-24 and miR-181b (Fig. 3A). Furthermore, the expression levels of miR-24 $(\mathrm{P}<0.0001)$ and miR-181b $(\mathrm{P}=0.0006)$ were both significantly increased in $3 \mu \mathrm{M}$ celastrol-treated A549 cells compared with the control treatment (Fig. 3B), indicating that celastrol may decrease the expression level of STAT3 via up-regulation of miR-24 and miR-181b. Additionally, LTEP-a-2 cells were also used to 
A
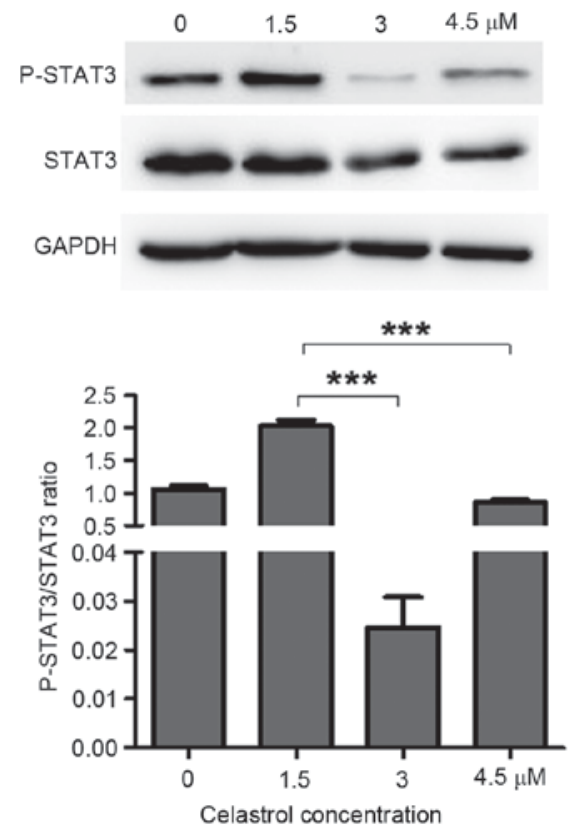
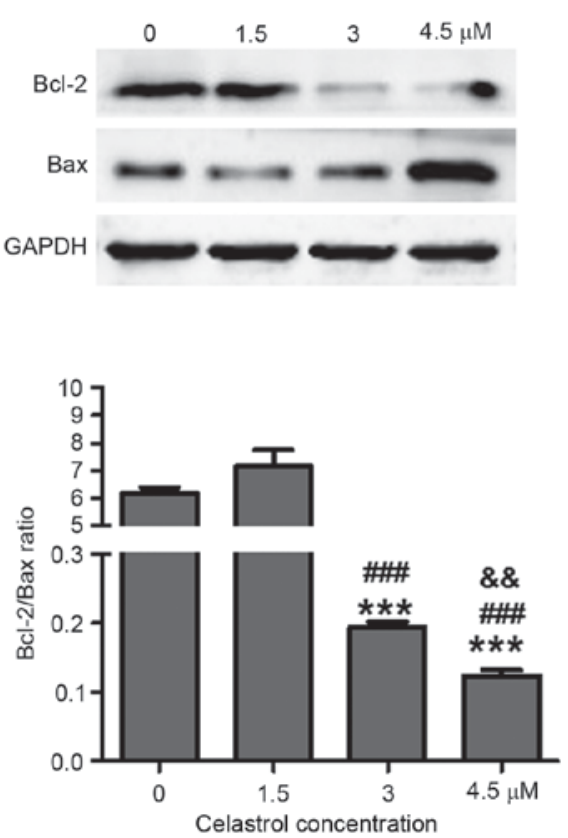

Figure 2. Celastrol inhibits the STAT3 signaling pathway and decreases the Bcl-2/Bax ratio. (A) Western blot analysis of STAT3 phosphorylation following celastrol treatments of 3 and $4.5 \mu \mathrm{M}$. STAT3 phosphorylation was significantly suppressed. $\mathrm{N}=3$ replicates. ${ }^{* * * *} \mathrm{P}<0.001,3 \mu \mathrm{M}$ vs. $1.5 \mu \mathrm{M} ;{ }^{* * *} \mathrm{P}<0.001,4.5 \mu \mathrm{M}$ vs. $1.5 \mu \mathrm{M}$ (B) Western blot analysis of the Bcl-2/Bax ration following treatment with celastrol. An increase in celastrol concentration significantly decreased the Bcl-2/Bax ratio in $\mathrm{A} 549$ cells. ${ }^{* * *} \mathrm{P}<0.001$ vs. $0 \mu \mathrm{M} ;{ }^{\# \# \#} \mathrm{P}<0.001$ vs. $1.5 \mu \mathrm{M} ;{ }^{\& \&} \mathrm{P}<0.01$ vs. $3 \mu \mathrm{M} . \mathrm{n}=3$ replicates. STAT3, signal transducer and activator of transcription 3; Bcl-2, B cell lymphoma-2; p, phosphorylation.

A

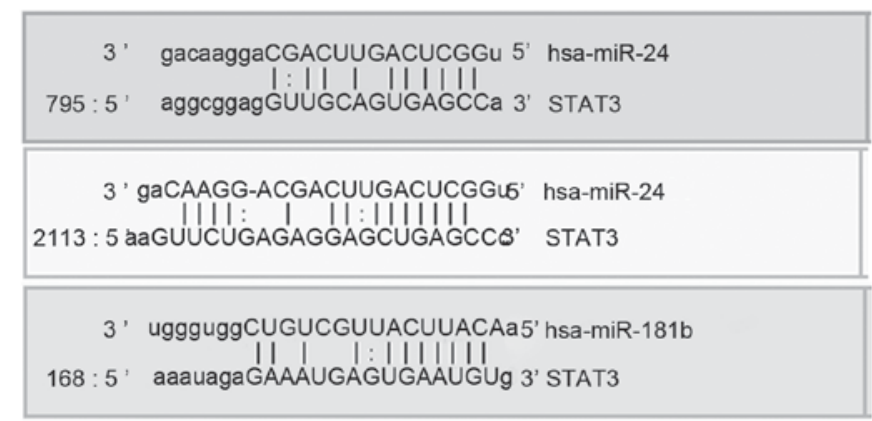
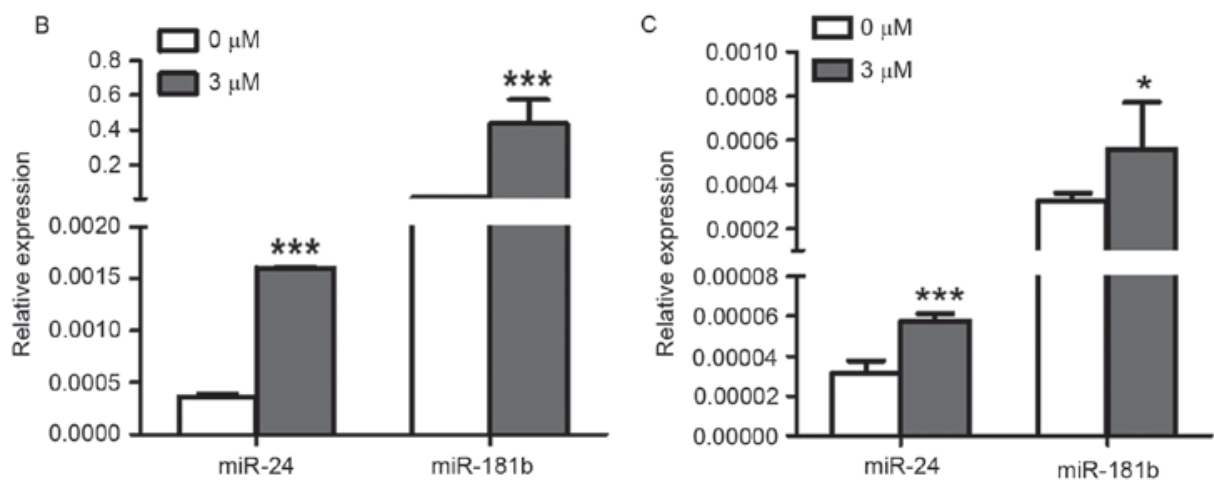

Figure 3. Celastrol treatment increases expression levels of miR-24 and miR-181b in A549 cells, and miR-24 and miR-181b target STAT3. (A) The targeting sites of STAT3, miR-24 and miR-181b are illustrated. Each miRNA is aligned with the mRNA of human STAT3 with the nucleotide position on the STAT3 mRNA indicated. Vertical lines indicate identity; gaps indicate mismatch. (B) Reverse transcription-quantitative polymerase chain analysis of miR-24 and miR-181b in A549 cells following treatment with celastrol. The expression levels of miR-24 and miR-181b were significantly increased in A549 cells treated by $3 \mu \mathrm{M}$ celastrol treatment. $\mathrm{n}=3$ replicates. (C) The expression levels of miR-24 and miR-181b were significantly increased in LETP-a- 2 cells treated with $3 \mu \mathrm{M}$ celastrol. $\mathrm{n}=3$ replicates. miR, microRNA; STAT3, signal transducer and activator of transcription 3 . ${ }^{*} \mathrm{P}<0.05$; ${ }^{* * *} \mathrm{P}<0.001$ compared with the $0 \mu \mathrm{M}$ control group.

confirm the effects of celastrol on the expression levels of miR-24 and miR-181b with $3 \mu \mathrm{M}$ celastrol stimulation. As expected, miR-24 ( $\mathrm{P}=0.0005)$ and miR-181b $(\mathrm{P}=0.03)$ were upregulated in celastrol-treated LTEP-a-2 cells (Fig. 3C). 
A

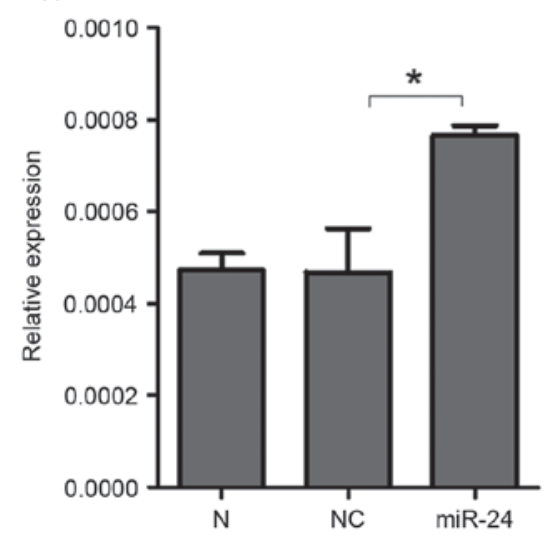

B

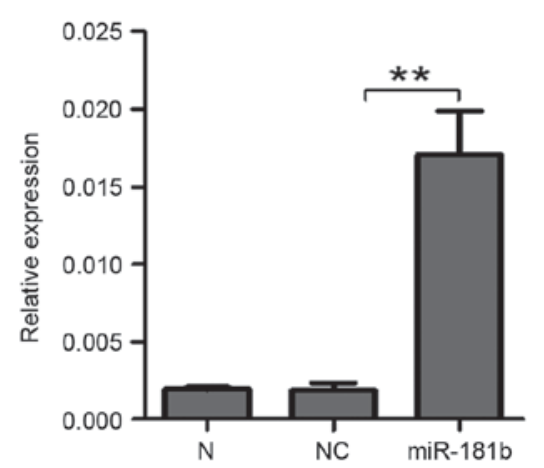

Figure 4. Treatment of A549 cells with miR-24 and -181b significantly increases their expression. Reverse transcription-quantitative polymerase chain reaction analysis of (A) miR-24 and (B) miR-181b expression levels. ${ }^{*} \mathrm{P}<0.05 ;{ }^{* *} \mathrm{P}<0.01$ compared with $\mathrm{NC}$ group. miR, microRNA. NC, negative control; $\mathrm{N}$, natural status prior to treatment.

A
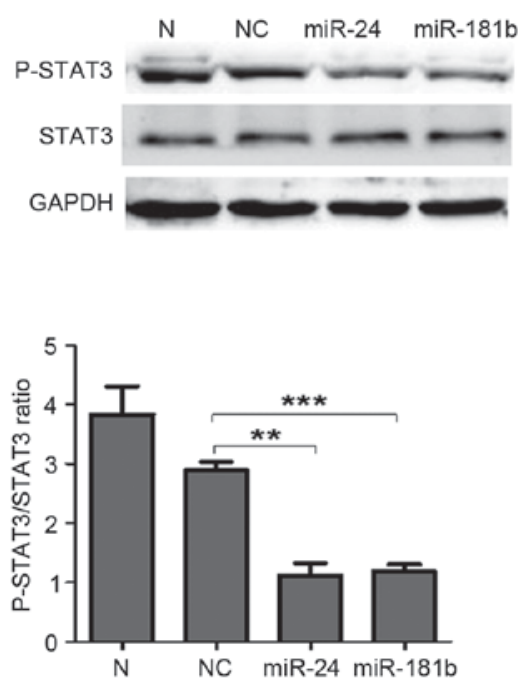

B
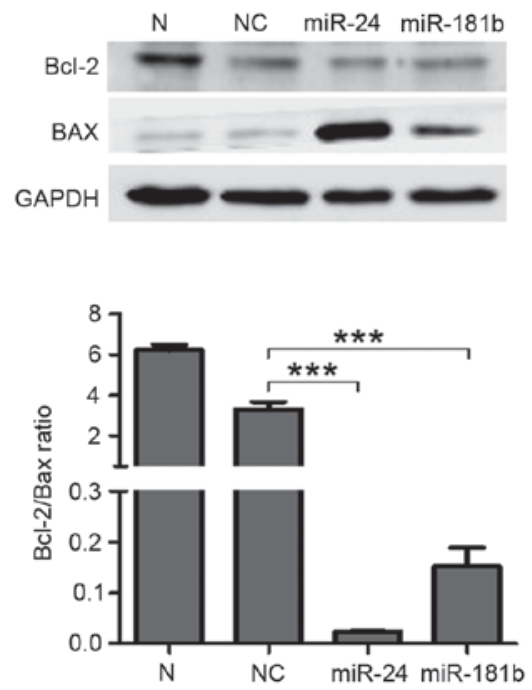

Figure 5. Treatment with miR-24 and miR-181b transfection inhibits STAT3 activation and decreases the Bcl-2/Bax ratio in A549 cells. (A) Western blot analysis of p-STAT3 expression levels following treatment with miR-24 and -181b. p-STAT3 expression levels were reduced in miR-24- and miR-181b-treated A549 cells compared with in the controls, whereas STAT3 expression level was not significantly affected by miR-24 and miR-181 treatment. $\mathrm{n}=3$ replicates. ${ }^{* *} \mathrm{P}<0.01 ;{ }^{* * *} \mathrm{P}<0.001$ compared with NC group. (B) Western blot analysis of the Bcl-2/Bax ratio. The Bcl-2/Bax ratio was significantly reduced with miR-24 and miR-181b treatment compared with NC treatment. $\mathrm{n}=3$ replicates. ${ }^{* * *} \mathrm{P}<0.001$ compared with NC group. miR, microRNA; STAT3, signal transducer and activator of transcription 3; Bcl-2, B cell lymphoma-2; p, phosphorylation; NC, negative control; N, natural status prior to treatment.

STAT3 phosphorylation and Bcl-2/Bax ratio is suppressed by miR-24 and miR-181b. To further investigate the effects of miR-24 and miR-181b on STAT3, synthesized miR-24 and miR-181b was transfected into A549 cells. RT-qPCR demonstrated that the expression levels of miR-24 ( $\mathrm{P}=0.04$, Fig. 4A) and miR-181b ( $\mathrm{P}=0.006$, Fig. 4B) significantly increased in the A549 cells treated with miR-24 and miR-181b compared with the negative control group, which indicated that miR-24 and miR-181b were successfully transfected into A549 cells. Western blotting revealed that miR-24 $(\mathrm{P}=0.002)$ and miR-181b $(\mathrm{P}=0.0007)$ treatment significantly suppressed the p-STAT3 levels compared with the negative control group (Fig. 5A). Furthermore, miR-24 ( $\mathrm{P}=0.0008)$ and miR-181b $(\mathrm{P}=0.0009)$ significantly decreased the expression ratio of $\mathrm{Bcl}-2$ and $\mathrm{Bax}$ compared with the negative control group $(\mathrm{P}<0.001$; Fig. 5B).
LTEP-a-2 cells were used to confirm these results. MiR-24 and miR-181b treatment significantly increased the level of miR-24 ( $\mathrm{P}=0.04$, Fig. 6A) and miR-181b ( $\mathrm{P}=0.04$, Fig. 6B) compared with the negative control group and significantly decreased p-STAT3 compared with the negative control group $(\mathrm{P}=0.04$ for miR-24 and $\mathrm{P}=0.04$ for miR-181b; Fig. 7A). In addition, miR-24 ( $\mathrm{P}=0.03$, Fig. 7B) and miR-181b ( $\mathrm{P}=0.006$, Fig. 7B) treatment significantly decreased the $\mathrm{Bcl}-2 / \mathrm{Bax}$ ratio compared with the control group in LTEP-a-2 cells. These results suggested that miR-24 and miR-181b mediated the effects of celastrol on the phosphorylation levels of STAT3 and the Bcl-2/Bax ratio.

\section{Discussion}

Previously, natural preparation represented a significant portion of the pharmaceutical market compared with 
A

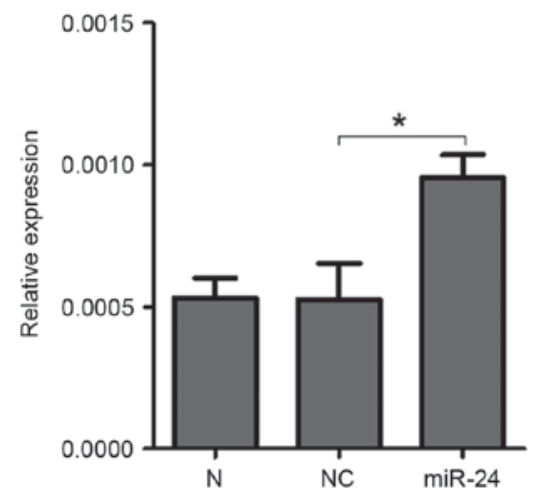

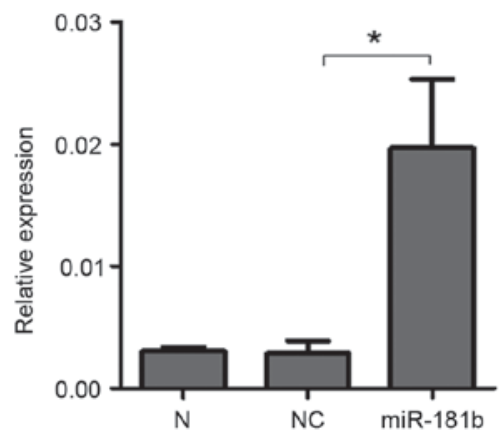

Figure 6. Treatment of LETP-a-2 cells with miR-24 and -181b significantly increases their expression. Reverse transcription-quantitative polymerase chain reaction analysis of (A) miR-24 and (B) miR-181b expression levels. " $\mathrm{P}<0.05$ compared with NC group. miR, microRNA; NC, negative control; N, natural status prior to treatment.

A
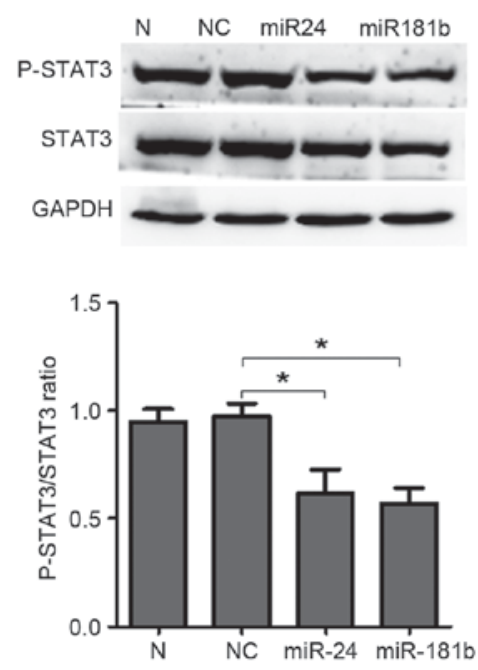

B
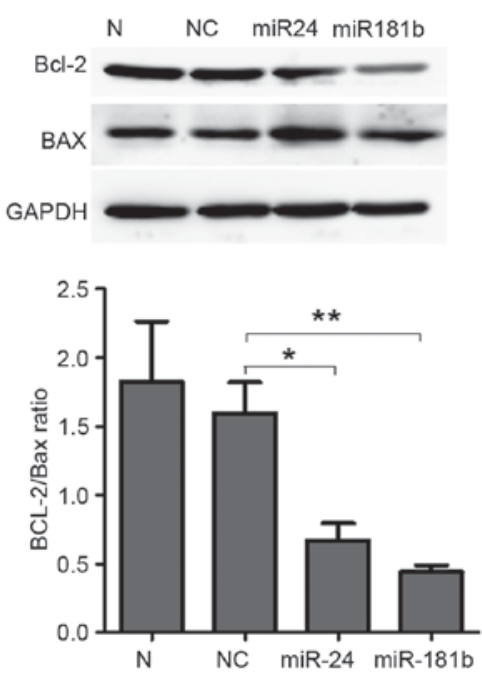

Figure 7. Treatment with miR-24 and miR-181b inhibits STAT3 activation and decreases the Bcl-2/Bax ratio in LETP-a-2 cells. (A) Western blot analysis of p-STAT3 expression levels. p-STAT3 expression level was markedly decreased in miR-24- and miR-181b-treated LETP-a-2 cells compared with the controls, whereas STAT3 expression level was not significantly affected by miR-24 and miR-181b treatment. $\mathrm{n}=3$ replicates. (B) Western blot analysis of the Bcl-2/Bax ratio. Bcl-2/Bax ratio was significantly reduced with miR-24 and miR-181b treatment compared with the $\mathrm{NC}$ treatment. $\mathrm{n}=3$ replicates. ${ }^{*} \mathrm{P}<0.05$; ${ }^{* *} \mathrm{P}<0.01$ compared with NC group. miR, microRNA; NC, negative control; N, natural status prior to treatment; STAT3, signal transducer and activator of transcription 3; Bcl-2, B cell lymphoma-2; p, phosphorylation.

synthesized compounds (2-4). Celastrol is extracted from Thunder God Vine, which contains a number of therapeutic active compounds (2). Since the 1970s, celastrol has been identified to possess anti-inflammatory, anti-oxidative and tumor cell-inducing apoptotic properties. Furthermore, compared with other anticancer drugs, celastrol selectively targets tumor cells and causes no obvious damage to normal hematopoietic cells, the heart, liver, kidney or other organs (20-22). Therefore, celastrol is considered to be a relatively safe and effective anticancer drug. Cumulative studies have identified numerous molecular targets of celastrol; however, studies investigating how celastrol affects these targets are rare. The present study treated the A549 lung adenocarcinoma cell line with various concentrations of celastrol to observe these effects. The results suggested that celastrol suppresses the proliferation and induces apoptosis of A549 cells in a dose-dependent manner. Of note, it was revealed that STAT3-associated miRNAs participated in the process of celastrol-mediated induction of A549 cell apoptosis.

STAT3 is a transcription factor that is phosphorylated by Janus kinases in response to cytokine activation (23). Subsequently, it dimerizes and translocates to the nucleus to activate transcription of cytokine-responsive genes (24). In tumorigenesis, STAT3 activation induces tumor progression by promoting the cell cycle and preventing apoptosis $(12,23)$. The present study demonstrated that celastrol induces cell apoptosis by decreasing the levels of STAT3 phosphorylation.

Furthermore, Bcl-2 and Bax have been reported to be downstream factors of STAT3, and they serve important roles in the mitochondrial-mediated apoptosis pathway $(18,20)$. $\mathrm{Bcl}-2$ is an anti-apoptotic protein and Bax is a pro-apoptotic protein. Apoptosis is largely controlled by the balance between anti-apoptotic and pro-apoptotic proteins, including the $\mathrm{Bcl}-2 / \mathrm{Bax}$ ratio. In the present study, celastrol significantly 
decreased STAT3 phosphorylation and the Bcl-2/Bax ratio, which supported the hypothesis that celastrol induces apoptosis by affecting the expression of STAT3 phosphorylation and the $\mathrm{Bcl}-2 / \mathrm{Bax}$ ratio.

Certain studies have demonstrated that celastrol may influence the expression levels of numerous miRNAs and achieve the therapeutic effects via these miRNAs $(15,16)$, which regulate a number of gene expression levels by targeting the 3'-UTR of mRNA molecules. To further investigate whether celastrol affected the expression of STAT3-associated miRNAs, miRNA analysis software was used to predict that the 3'-UTR of STAT3 was targeted by miR-24 and miR-181b. Previous studies indicated that miR-24 may regulate liver inflammation in rats and that miR-181b functions as a tumor suppressor in glioma $(25,26)$. Of note, the present study revealed that $\mathrm{p}$-STAT3 was significantly reduced by miR-24 and miR-181b. In addition, the Bcl-2/Bax ratio also decreased in miR-24 and miR-181b-treated cells. These results confirmed that miR-24 and miR-181b may influence STAT3 activation (27). Furthermore, celastrol treatment increased the expression levels of miR-24 and miR-181b, and decreased STAT3 phosphorylation and the $\mathrm{Bcl}-2 / \mathrm{Bax}$ ratio. These results indicated that celastrol may decrease p-STAT3 levels by regulating miR-24 and miR-181b.

In conclusion, the present study investigated the molecular mechanism underlying celastrol in lung cancer therapy. It was demonstrated that celastrol induced apoptosis of lung cancer cells by affecting the expression levels of miR-24 and miR-181b, which further regulated the activation of STAT3. Identification of the function of miR-24 and miR-181b provides useful information for the safe and effective application of celastrol in tumor therapy.

\section{Acknowledgements}

The present study was supported by the National Natural Science Foundation (grant nos. 31371321, 31440061 and 81530060), and the Shandong Science and Technology Committee (grant nos. ZR2014HP004, 2015GSF118073, ZR2014HL055 and ZR2013HL003), the Health and Family Planning Commission of Shandong Province (grant no. 2014WS0185) and the Shandong Education Department (grant no. J13LE11).

\section{References}

1. Salminen A, Lehtonen M, Paimela T and Kaarniranta K Celastrol: Molecular targets of thunder god vine. Biochem Biophys Res Commun 394: 439-442, 2010.

2. Li-Weber M: Targeting apoptosis pathways in cancer by Chinese medicine. Cancer Lett 332: 304-312, 2013.

3. Corson TW and Crews CM: Molecular understanding and modern application of traditional medicines: Triumphs and trials. Cell 130: 769-774, 2007.

4. Patwardhan B and Mashelkar RA: Traditional medicine-inspired approaches to drug discovery: Can Ayurveda show the way forward?. Drug Discov Today 14: 804-811, 2009.

5. Koehn FE: High impact technologies for natural products screening. Prog Drug Res 65: 175, 177-210, 2008.

6. Lipsky PE and Tao XL: A potential new treatment for rheumatoid arthritis: Thunder god vine. Semin Arthritis Rheum 26: 713-723, 1997.
7. Canter PH, Lee HS and Ernst E: A systematic review of randomised clinical trials of Tripterygium wilfordii for rheumatoid arthritis. Phytomedicine 13: 371-377, 2006.

8. Petronelli APannitteri GTesta UTriterpenoids as new promising anticancer drugs. Anticancer Drugs 20: 880-892, 2009.

9. Sethi G, Ahn KS, Pandey MK and Aggarwal BB: Celastrol, a novel triterpene, potentiates TNF-induced apoptosis and suppresses invasion of tumor cells by inhibiting NF-kappaB-regulated gene products and TAK1-mediated NF-kappaB activation. Blood 109: 2727-2735, 2007.

10. Yang H, Chen D, Cui QC, Yuan X and Dou QP: Celastrol, a triterpene extracted from the Chinese 'Thunder of God Vine,' is a potent proteasome inhibitor and suppresses human prostate cancer growth in nude mice. Cancer Res 66: 4758-4765, 2006.

11. Chen M, Rose AE, Doudican N, Osman I and Orlow SJ: Celastrol synergistically enhances temozolomide cytotoxicity in melanoma cells. Mol Cancer Res 7: 1946-1953, 2009.

12. Dai Y, DeSano JT, Meng Y, Ji Q, Ljungman M, Lawrence TS and $\mathrm{Xu}$ L: Celastrol potentiates radiotherapy by impairment of DNA damage processing in human prostate cancer. Int J Radiat Oncol Biol Phys 74: 1217-1225, 2009.

13. Rajendran P, Li F, Shanmugam MK, Kannaiyan R, Goh JN, Wong KF, Wang W, Khin E, Tergaonkar V, Kumar AP, et al: Celastrol suppresses growth and induces apoptosis of human hepatocellular carcinoma through the modulation of STAT3/JAK2 signaling cascade in vitro and in vivo. Cancer Prev Res (Phila) 5: 631-643, 2012.

14. Costantino L and Barlocco D: STAT 3 as a target for cancer drug discovery. Curr Med Chem 15: 834-843, 2008.

15. Sha M, Ye J, Zhang LX, Luan ZY, Chen YB and Huang JX: Celastrol induces apoptosis of gastric cancer cells by miR-21 inhibiting PI3K/Akt-NF- $\mathrm{BB}$ signaling pathway. Pharmacology 93: 39-46, 2014.

16. Li H, Li Y, Liu D, Sun H and Liu J: MiR-224 is critical for celastrol-induced inhibition of migration and invasion of hepatocellular carcinoma cells. Cell Physiol Biochem 32: 448-458, 2013.

17. Wang PY, Sun YX, Zhang S, Pang M, Zhang HH, Gao SY, Zhang C, Lv CJ and Xie SY: Let-7c inhibits A549 cell proliferation through oncogenic TRIB2 related factors. FEBS Lett 587: 2675-26812013.

18. You S, Li R, Park D, Xie M, Sica GL, Cao Y, Xiao ZQ and Deng X: Disruption of STAT3 by niclosamide reverses radioresistance of human lung cancer. Mol Cancer Ther 13: 606-616, 2014.

19. Livak KJ and Schmittgen TD: Analysis of relative gene expression data using real-time quantitative PCR and the 2(-Delta Delta C(T)) method. Methods 25: 402-408, 2001.

20. Real PJ, Sierra A, De Juan A, Segovia JC, Lopez-Vega JM and Fernandez-Luna JL: Resistance to chemotherapy via Stat3-dependent overexpression of Bcl-2 in metastatic breast cancer cells. Oncogene 21: 7611-7618, 2002.

21. Zhang C, Chi YL, Wang PY, Wang YQ, Zhang YX, Deng J, Lv CJ and Xie SY: miR-511 and miR-1297 inhibit human lung adenocarcinoma cell proliferation by targeting oncogene TRIB2. PLoS One 7: e46090, 2012.

22. Li YJ, Zhang YX, Wang PY, Chi YL, Zhang C, Ma Y, Lv CJ and Xie SY: Regression of A549 lung cancer tumors by anti-miR-150 vector. Oncol Rep 27: 129-134, 2012.

23. Ji N, Li J, Wei ZKong F, Jin H, Chen X, Li Y and Deng Y: Effect of celastrol on growth inhibition of prostate cancer cells through the regulation of hERG channel in vitro. Biomed Res Int 2015: 308475,2015

24. DiSanto JP: Cytokines: Shared receptors, distinct functions. Curr Biol 7: R424-R426, 1997.

25. Dogan I, Cumaoglu A, AriciogluA and EkmekciA: Inhibition of ErbB2 by herceptin reduces viability and survival, induces apoptosis and oxidative stress in Calu-3 cell line. Mol Cell Biochem 347: 41-51, 2011.

26. Tian SS, Lamb P, Seidel HM, Stein RBRosen J: Rapid activation of the STAT3 transcription factor by granulocyte colony-stimulating factor. Blood 84: 1760-1764, 1994.

27. Cao Q, Li YY, He WF, Zhang ZZ, Zhou Q, Liu X, Shen Y and Huang TTInterplay between microRNAs and the STAT3 signaling pathway in human cancers. Physiol Genomics 45: 1206-1214, 2013 